\title{
Aspectos clínicos e macroscópicos da palatoplastia imediata com implante de cartilagem da pina articular, conservada em glicerina a $98 \%$, após indução experimental de fenda palatina em cães
}

\author{
Clinic and macroscopic view of immediate palatoplasty with auricular pinna \\ cartilage preserved in $98 \%$ glycerin in canine experimental palat cleft
}

\author{
Emerson Antônio Contesini ${ }^{1}$ Ney Luis Pippi ${ }^{2}$ Carlos Afonso de Castro Beck $^{1}$ \\ Maurício Veloso Brun ${ }^{1}$ Marshal da Costa Leme ${ }^{1}$ Alceu Gaspar Raiser ${ }^{3}$ \\ Luis Carlos de Pellegrini ${ }^{4}$ Adamas Tassinari Bonfada ${ }^{5}$ Thiago Félix da Silva ${ }^{5}$ \\ Jane Stella Cândido da Costa ${ }^{5}$ Anelise Bonilla Trindade ${ }^{5}$ Evandro Pezzini França $^{5}$
}

\section{RESUMO}

Os defeitos de espessura completa do palato que resultam em comunicação oro-nasal raramente cicatrizam espontaneamente. Eles requerem reparo cirúrgico, porém, pode ser dificil obter cicatrização satisfatória devido às condições próprias das cavidades nasal e oral. Neste experimento, foram utilizados 14 cães, reunidos em dois grupos de igual número. Todos animais foram submetidos à indução de fenda palatina experimental. $O$ grupo $G 1$ foi o controle, cuja palatoplastia foi realizada a partir da confecção de "flap" muco-periósteo. No grupo G2, foi utilizada cartilagem da pina auricular conservada em glicerina a 98\% entre o osso palatino e o muco-periósteo. Os animais foram avaliados macroscopicamente quanto ao processo cicatricial, por radiografias para a regeneração óssea e por rinoscopia para observar a regeneração da mucosa nasal. Foi observada a cicatrização da mucosa oral a partir do $10^{\circ}$ dia, com deiscência de sutura em dois animais do grupo G1. A radiografia demonstrou evolução na cicatrização óssea, num processo mais acelerado no grupo G2 quando comparado ao grupo G1. A rinoscopia revelou, aos 60 dias, completa regeneração da mucosa nasal em ambos os grupos. Ao final do periodo de avaliação foi observada macroscopicamente, completa fusão óssea nos animais do grupo G2 e incompleta nos do grupo G1, o que demonstrou a interferência benéfica do implante no processo cicatricial do grupo $G 2$.

\section{ABSTRACT}

The incomplete defects of hard palate result in oronasal fistula seldom cure themselves in a spontaneous way, due to limited condition in oral and nasal cavity it may be difficult to obtain satisfactory cicatrization, requiring surgery to interfere in the healing process. Fourteen dogs arranged in two groups were submitted to experimental hard palate cleft. The G1 group was the control group, in which palatoplasty was achieved with mucous-periosteum flap. In the G2 group, a preserved at $98 \%$ glycerin auricular pinna cartilage was used between the palate bone and mucous-periosteum. The mucosal healing and bone regeneration were evaluated, radiographied and the nasal mucosa was observed by rhinoscopy. The oral mucosa heal was observed at the tenth day, but the suture dehiscence occurred in two dogs from the G1 group. The radiography evaluation demonstrated bone regeneration evolution quicker in the G2 group, than in the G1 group. The complete nasal mucous regeneration was demonstrated by rhinoscopy at the sixtieth day. The conclusive evaluation observed full bone union in the G2 group and unachieved bone union in the G1 group, which showed graft interference in the $G 2$ group bone regeneration.

Key words: palatoplasty, graft, glycerin, canine.

Palavras-chave: palatoplastia, implante, glicerina, cães

\footnotetext{
${ }^{1}$ Médico Veterinário, Professor Assistente, Departamento de Clínica Médica Veterinária, Universidade Federal de Mato Grosso, Doutorando em Cirurgia Experimental na Universidade Federal de Santa Maria (UFSM), Camobi, 97105-900, Santa Maria, RS. E-mail: eacont@terra.com.br. Autor para correspondência.

${ }^{2}$ Médico Veterinário, PhD., Professor Adjunto, Departamento de Clínica de Pequenos Animais (DCPA), Centro Ciências Rurais (CCR), UFSM

${ }^{3}$ Médico Veterinário, Doutor, Professor Titular, DCPA, CCR, UFSM.

${ }^{4}$ Médico Veterinário, Professor Adjunto, Departamento de Clínica de Grandes Animais, CCR, UFSM.

${ }^{5}$ Acadêmicos do Curso de Graduação em Medicina Veterinária, CCR, UFSM.
} 


\section{INTRODUÇÃO}

A oclusão dos defeitos palatinos em neonatos é repleta de problemas como: a ocorrência de tensão na linha de sutura, as tensões do crescimento, a falta de tecido disponível, a impossibilidade de imobilização dos tecidos em decorrência dos movimentos da língua e respiratórios, levando à deiscência. A cicatrização de grandes áreas deixadas abertas pelo desvio de retalhos muco-periostais palatinos provoca anormalidades do crescimento do palato, devido à contração do colágeno no tecido de granulação junto à arcada dentária (NELSON, 1998).

A fenda palatina adquirida tem como principais etiologias aquelas relacionadas com traumas como lacerações por mordidas caninas, arma de fogo, fios de condução de eletricidade, infecções crônicas graves, terapia cirúrgica e por radiação (HARVEY, 1987). Também são citadas as neoplasias como mastocitoma, epúlide fibromatoso, fibrossarcoma (HEAD, 1990); tumores malignos indiferenciados, fibromeloblastoma em gatos (WITHROW, 1996) e osteossarcoma (BECK \& STRIZEK, 1999).

Os sinais clínicos associados ao palato fendido variam com o grau do defeito e podem incluir crescimento insatisfatório, drenagem de leite pelas narinas durante e após a amamentação, em animais jovens; ainda tosse, esforços para vomitar, espirros durante a alimentação e infecções recidivantes do trato respiratório (HOSKINS \& DIMSKI, 1997).

Entre os principais problemas encontrados na rinoscopia de pequenos animais, estão o pequeno calibre das vias nasais, a frágil natureza dos ossos turbinados e da membrana mucosa. Sua indicação é principalmente quando há necessidade de inspeção e acesso à mucosa, com base na anamnese clínica e em radiografias nasais (ORTON \& PARK, 1998).

Segundo a publicação de HARVEY (1987), vários materiais de sutura têm sido utilizados com sucesso na cavidade oral. Se os nós são deixados na superfície epitelial, geralmente tanto as suturas absorvíveis como as inabsorvíveis são liberadas espontaneamente com três ou quatro semanas. A preferência do material recai sobre os materiais absorvíveis sintéticos, utilizados em padrões alternando suturas interrompidas verticais com suturas simples separadas. Isso permite maior inclusão de tecido conectivo quando comparado com uso de apenas pontos simples separados.

Existem diversas técnicas para correção de fenda palatina congênita. SALISBURY (1996) e NELSON (1998) sugeriram o fechamento em duas camadas, uma feita pela mucosa nasal, outra pelo muco- periósteo oral. A técnica sugerida por HARVEY (1987), para o reparo de fenda palatina congênita medial envolve a sobreposição de "flaps"; nas fendas adquiridas, o uso de "flap" muco-periosteal. Geralmente são utilizados "flaps" do tipo rotação ou de deslizamento para recobrir uma grande área, pois permitem cobertura maior. Também SAGER \& NEFEN (1998) descreveram uma técnica de confecção de "flap" por deslizamento de mucosa bucal do palato mole. A utilização da língua como sítio doador para a confecção de "flap" foi descrita por BUSIC et al. (1989), e posteriormente por ASSUNÇÃO (1993), como alternativa para correção de grandes defeitos do palato duro em humanos.

FUTRAN \& HALLER (1999) publicaram relato da transferência microvascular de tecido livre como alternativa na reconstrução de defeitos palatinos subtotal e total, além de hemipalato. Uma mistura adequada de osso e/ou tecido mole pode ser transferida num único estágio.

Objetivou-se neste experimento, testar uma nova alternativa para o padrão de sutura palatina, avaliar o emprego de "flap" de muco-periósteo por deslizamento na obliteração da fenda palatina, utilizar a cartilagem da pina auricular conservada em glicerina a $98 \%$, como alternativa na redução de fenda de palato duro em cães, investigando seu favorecimento no reparo ósseo.

\section{MATERIAL E MÉTODOS}

Este experimento foi desenvolvido no Laboratório de Cirurgia Experimental da Universidade Federal de Santa Maria, utilizando 14 cães, sem raça definida, nem distinção de sexo, adultos hígidos, com peso médio de $15 \mathrm{~kg}$, oriundos do Biotério Central dessa Universidade. Os animais foram submetidos a exame físico, everminados e alojados em canis individuais, receberam ração comercial e água ad libitum. O experimento envolveu dois grupos, sendo todos os animais submetidos à indução experimental de fenda palatina com tamanho médio de $3,5 \times 1,5 \mathrm{~cm}$ e a palatoplastia realizada imediatamente após a indução. No grupo G1 (controle) os cães foram submetidos à redução da fenda palatina com a confecção de "flap" por deslizamento do muco-periósteo e, no grupo G2, os cães foram submetidos à redução da fenda palatina semelhante ao grupo anterior, porém com cartilagem da pina auricular homóloga, conservada em glicerina a $98 \%$, implantada entre o osso palatino e o mucoperiósteo.

Utilizou-se o protocolo anestésico composto por maleato de acepromazina $\left(0,1 \mathrm{mg} \cdot \mathrm{kg}^{-1}, \mathrm{IV}\right)$ e citrato 
de fentanila $\left(0,005 \mathrm{mg} \cdot \mathrm{kg}^{-1}, \mathrm{IV}\right)$ como medicação préanestésica. $\mathrm{Na}$ indução da anestesia geral foi utilizado o tiopental sódico $\left(12,5 \mathrm{mg} \cdot \mathrm{kg}^{-1}, \mathrm{IV}\right)$ e a manutenção, com halotano vaporizado em $\mathrm{O}_{2}$ a $100 \%$ com circuito semi-aberto.

A cartilagem foi mantida submersa em solução de glicerina a $98 \%$, durante um período mínimo de 30 dias. Vinte e quatro horas antes da cirurgia, a cartilagem foi removida desse meio conservante e mantida em solução de PVP-I diluído em solução de $\mathrm{NaCl} 0,9 \%$, numa proporção de 1:50. No momento da implantação, foi lavada com $\mathrm{NaCl} 0,9 \%$.

O decúbito utilizado foi o dorsal, com a boca mantida aberta com auxílio de abridor de bocas para cães. A anti-sepsia da cavidade oral foi realizada com gaze cirúrgica embebida em solução de clorexidine a $0,2 \%$, utilizou-se ainda a gaze cirúrgica dobrada, posicionada na região da laringe.

O uso de antibiótico foi profilático à confecção da fenda palatina, com ampicilina sódica (20mg.kg${ }^{1}$ IV), 30 minutos antes do início da cirurgia. Os animais receberam flunixin meglunine no trans-operatório e nos dois dias subseqüentes ao mesmo, a cada 24 horas, na dose de $1,0 \mathrm{mg} \cdot \mathrm{kg}^{-1}$, IV.

A confecção da fenda palatina foi realizada a partir de uma incisão com bisturi de formato elíptico do muco-periósteo na rafe palatina de aproximadamente $4,5 \times 1,5 \mathrm{~cm}$. As margens incisadas do muco-periósteo foram elevadas do osso palatino com elevador de periósteo, lateralmente em direção aos dentes, sendo aplicados a seguir, dois pontos de reparo em cada borda muco-perióstea para visualização e acesso ao osso palatino. Na seqüência, foi removido um retalho longitudinal de osso palatino com dimensões aproximadas de $3,5 \times 1,5 \mathrm{~cm}$, com auxílio de uma serra vibratória, , efetivando a confecção da fenda palatina. A padronização das bordas sob formato retangular foi feita com auxílio de pinça goiva.

A técnica cirúrgica utilizada para a palatoplastia dos animais do grupo G1, foi efetuada a partir de duas incisões paralelas longitudinais no mucoperiósteo às margens do palato duro, imediatamente medial à arcada dentária, em toda extensão da fenda, respeitando-se a localização anatômica das artérias palatinas incluindo-as na confecção do "flap" bipediculado, permitindo a coaptação das bordas mucoperiosteais por deslizamento. A síntese para manutenção da coaptação entre as bordas foi realizada com fio de náilon monofilamentar 3-0, no padrão de sutura longe-perto-perto-longe.

No grupo G2, o implante de cartilagem da pina auricular, após receber o tratamento de reidratação e anti-sepsia, foi recortado em formato elíptico em di- mensões em torno de 5 a $6 \mathrm{~mm}$ maiores que as da fenda óssea, possibilitando seu perfeito encaixe entre as margens do defeito ósseo e as bordas do muco-periósteo palatino (Figura 1). Após, foi aplicada a mesma técnica descrita para o grupo G1.

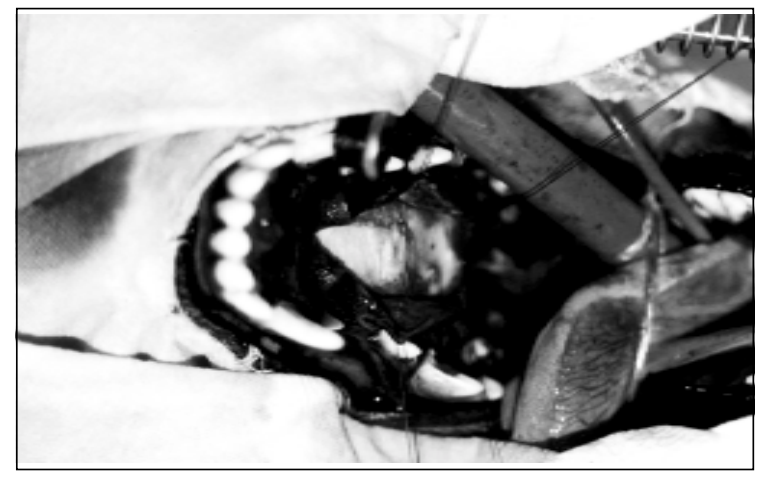

Figura 1 - Implante de cartilagem entre o muco-periósteo e o osso palatino em um cão do grupo G2 .

A dieta alimentar no período pós-operatório constou de água ad libitum e ração comercial. Nos primeiros quinze dias, a ração foi pastosa, triturada com água. A partir do décimo sexto dia foi fornecida ração seca. O pós-operátório imediato foi constituído por limpeza local com solução de clorexidine a $0,2 \%$ duas vezes ao dia, com avaliação diária da cicatrização da mucosa oral. Ao final de 10 dias, os pontos de sutura foram removidos. A cada quinze dias, os cães foram encaminhados para avaliação radiológica, para acompanhamento da evolução da regeneração óssea. Com sessenta dias de operados, realizou-se exame rinoscópico para avaliar a cicatrização da mucosa nasal.

Após 120 dias, os cães foram submetidos à eutanásia, para avaliação macroscópica e obtenção de material para posterior estudo histopatológico que será publicado noutro artigo, oportunamente.

\section{RESULTADOS E DISCUSSÃO}

A ingestão de ração pastosa nos primeiros 15 dias, foi considerada clinicamente normal, sem quaisquer manifestações de desconforto. A exceção ocorreu com dois animais do grupo G1, que apresentaram no $5^{\circ}$ (um deles) e $6^{\circ}$ (o outro) dias de pós-operatório (PO), sinais de tosse e regurgitação por via nasal, quando ao exame direto do local da cirurgia, foi observada deiscência de sutura nos terços caudal num animal e médio no outro, ambos de aproximadamente $1,5 \mathrm{~cm}$ de comprimento. A deiscência de sutura, considerada por HARVEY (1987) como uma das principais complica-

Ciência Rural, v. 33, n. 1, jan-fev, 2003. 
ções pós-operatórias, pode ser atribuída à fragilidade local, uma vez que os animais do grupo G1 não receberam o reforço da cartilagem. Outra hipótese pode ser decorrente da contração cicatricial das linhas de relaxamento, realizadas paralelas ao defeito do palato, ou ainda ao excesso de tensão no local da sutura por insuficiência no relaxamento local, fatos citados por HARVEY (1987) e por SALISBURY (1996), como causas do insucesso cirúrgico. A adaptação ao consumo de ração seca a partir do $16^{\circ}$ foi adequada, sem que os animais apresentassem dificuldade na preensão e deglutição. A ingestão de água foi normal durante todo o período de observação.

Os doze animais restantes apresentaram, ao $10^{\circ}$ dia de $\mathrm{PO}$, excelente cicatrização da mucosa oral e ainda era evidente a existência da maioria dos fios de sutura. Tal ocorrência já era esperada porque foi utilizado fio de náilon monofilamentar, o qual é inabsorvível, porém a movimentação contínua da língua removeu alguns deles. O restante foi removido nesse dia. Este resultado corrobora as afirmações de SALISBURY (1996), que classificou o fio mononáilon como um dos mais apropriados para a síntese da mucosa, devido à sua propriedade monofilamentar que confere a impermeabilidade necessária; concorda-se, ainda, com as de HARVEY (1987), o qual afirmou que fios absorvíveis e não absorvíveis podem ser utilizados, pois a tendência é de haver liberação espontânea dos mesmos, desde que os nós sejam mantidos na superfície epitelial.

A excelente capacidade cicatricial da mucosa palatina foi verificada pela cicatrização das linhas de relaxamento paralelas à fenda, mesmo tendo sido bem afastadas em suas bordas para o deslizamento dos "flaps". Tal qualidade de cicatrização foi favorecida por um grau de afastamento que não comprometeu a irrigação sangüínea. Uma atenção especial foi dedicada à irrigação do "flap", uma vez que NELSON (1998) relatou a dificuldade que alguns métodos de oclusão de defeitos palatinos apresentam na cicatrização do muco-periósteo, quando grandes áreas permanecem descobertas pelo desvio de retalhos mucoperiósteos.

A utilização da sutura longe-perto-perto-longe, conferiu ancoragem adequada para o fio, sem comprometer o aporte sangüíneo local, uma vez que apresenta quatro pontos de reforço. Esse padrão de sutura parece apresentar vantagens sobre os descritos por HARVEY (1987) e BECK \& STRIZEK (1999), que são o simples interrompido e o padrão de Wolf. Sua elaboração, de fácil aplicação, possibilita maior número de pontos de ancoragem em relação ao simples interrompido, produzindo a mesma qualidade de coaptação, porém com maior reforço. Quando comparado ao ponto de Wolf, tem a vantagem de evitar a evaginação o que envolve a utilização de maior quantidade de tecido, acarretando maior deslocamento do "flap" e, conseqüentemente, aumentando a distância entre as bordas nas incisões de relaxamento. Seu emprego apresenta uma alternativa inovadora neste tipo de cirurgia, pois a literatura consultada não abordou o seu uso.

A obliteração da fenda palatina foi de fácil realização, em decorrência da utilização de "flap" por deslizamento associado às duas incisões paralelas de relaxamento, semelhante às afirmações feitas por LAMMERDING et al. (1976), HARVEY (1987) e SAGER \& NEFEN (1998). Porém não se enquadra nas afirmações feitas por TOUFFUT et al. (1993) que consideraram as opções cirúrgicas, exceto a colocação de agrafes, como sendo alternativas de difícil execução e pouco confiáveis. Esse procedimento permitiu o deslocamento tecidual sem tensão, não interferindo na vascularização, uma vez que as artérias palatinas, foram deslocadas juntamente com os "flaps". Tal procedimento corrobora as citações de SALISBURY (1996) que defendeu a incorporação dessas artérias para que haja a "pega" dos "flaps". Também a ausência de tensão na área de síntese foi fundamental para que não houvesse índice significativo de deiscência, fator considerado por HARVEY \& EMILY (1993) como essencial para o sucesso desse tipo de procedimento.

A exploração rinoscópica foi realizada aos 60 dias de cirurgia, sendo considerado um procedimento de fácil execução, não ocorrendo episódios de hemorragias como aqueles descritos por ORTON \& PARK (1998), requerendo apenas tranqüilização dos pacientes. As imagens demonstraram completa regeneração da mucosa nasal, evidenciando a presença de elevações relativamente desordenadas na mucosa. O uso desse tipo de exploração contribuiu de forma significativa para a avaliação da cicatrização da mucosa nasal, pois não haveria acesso a essas imagens por visualização direta, para verificar a reparação na cavidade nasal.

O exame radiográfico realizado quinzenalmente, por período de 120 dias, demonstrou evolução satisfatória da regeneração óssea, com tempo diferenciado entre ambos os grupos, contudo homogênea dentro de cada um deles. Os animais do grupo G1 mostraram evolução mais lenta no processo cicatricial. Até o $45^{\circ}$ dia, as imagens radiográficas sugeriram a possibilidade de um processo inflamatório, com possível presença de líquido na região dos turbinados e escassa regeneração óssea. A partir de 60 dias, o processo in- 
flamatório começou a apresentar regressão e o líquido diminuiu significativamente. Aos 90 dias, as imagens radiográficas foram de completo desaparecimento desses sinais. No final de 120 dias, as radiografias mostraram processo de cicatrização óssea com pequenas falhas com largura média de 3-4mm para a coaptação óssea. Os animais do grupo G2 apresentaram cicatrização mais breve, além da ausência de líquido e a reação inflamatória dos tecidos moles esteve evidente apenas até 45 dias de PO, nas imagens radiográficas. Ainda, o processo de redução da falha óssea na fenda palatina foi mais rápido, não apresentando quaisquer sinais de espaço entre as bordas do defeito induzido. Aos 90 dias, todos os animais desse grupo apresentaram imagens compatíveis com completa regeneração óssea. Esses achados são compartilhados com os de SKOOG (1985) que alegou ser noventa dias o prazo mínimo necessário para obter oclusão completa de fenda palatina.

Ao final do período de 120 dias, na inspeção direta, durante a necrópsia, observou-se completa regeneração das mucosas oral e nasal.

Para que fosse possível averiguar a compatibilidade entre os achados macroscópicos e as imagens do estudo radiográfico, constatando existência do fechamento das bordas ósseas (Figura 2), foi introduzida uma agulha hipodérmica de insulina $(16 \times 6 \mathrm{G})$, a partir da mucosa oral na região medial na linha central do palato, a qual revelou, nos animais do grupo G1, presença de tecido de consistência fibrosa. Com a passa-

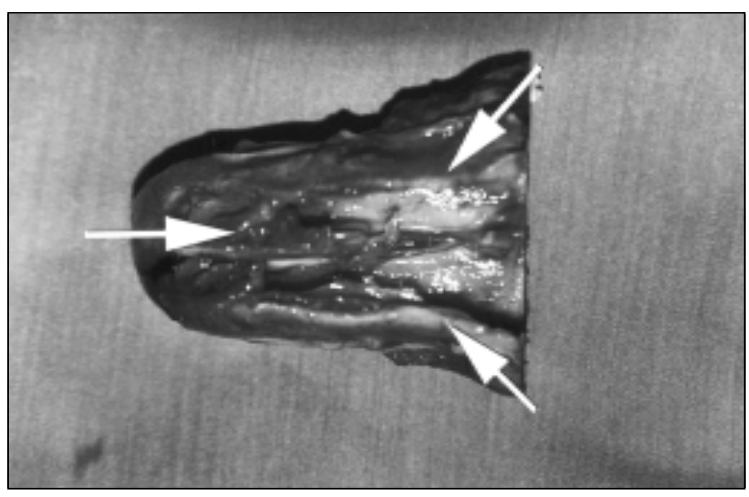

Figura 2 - Aspecto macroscópico da área de regeneração óssea de um cão do grupo G2, após 120 dias de reparo com implante de cartilagem (vista nasal).

gem da agulha, atravessando todo o tecido com alguma resistência, exteriorizando-se pela mucosa nasal; essas perfurações foram feitas para identificar o distanciamento entre as bordas do osso palatino. Nesse grupo, o distanciamento médio foi de $2-3 \mathrm{~mm}$, o que sugere fase final de regeneração. Nos animais do grupo G2, não foi possível exteriorizar a agulha pela mucosa nasal. A mesma, ao ser introduzida pela mucosa oral, sofreu resistência local, evidenciando um tecido de consistência óssea, rangendo ao toque de ponta da agulha, confirmando assim, as imagens radiográficas de completa regeneração do osso palatino. Observouse ainda, a presença de tecido de consistência firme, que se assemelhava a tecido fibroso, depositado entre as duas camadas de mucosa; esse tecido foi mais evidente nos animais do grupo G2. A diferença no comportamento cicatricial ósseo entre os dois grupos sugere que a presença do implante de cartilagem favorece a regeneração. Esses achados estão em acordo com os relatados por DIXIT et al. (1995) que, ao promoverem fenda palatina em cães, para pesquisar as propriedades osteogênicas do periósteo, obtiveram ilhas de crescimento ósseo a partir da insinuação do vômer juntamente com tecido de granulação, ocupando espaço entre as bordas ósseas em regeneração. Tal achado fez com que os autores concluíssem que, a partir da presença de tecido, associado ou não à reação fibrosa, interligando ambas as bordas, seria possível se obter um processo cicatricial ósseo mais rápido, uma vez que o fator de cicatrização do mesmo parece estar relacionado com o sucesso do fechamento cicatricial do defeito com tecido mole.

\section{CONCLUSÃO}

Os resultados obtidos permitem concluir que o "flap" de muco-periósteo por deslizamento é adequado para o fechamento de defeitos palatinos de $3,5 \times 1,5 \mathrm{~cm}$ no cão; o padrão de sutura longe-perto-perto-longe, é de fácil execução e promove coaptação com vedação adequada do palato e o uso do implante de cartilagem da pina auricular favorece a reparação mais precoce do osso palatino.

\section{REFERÊNCIAS BIBLIOGRÁFICAS}

ASSUNÇÃO, A.G. The design of tongue flaps for the closure of palatal fistulas. Journal of Plastic and Reconstructive Surgery, Campinas, v. 91, n. 5, p. 806-810, 1993.

BECK, J.A.; STRIZEK, A.A. Full-thickness resection of the hard palate for treatment of osteosarcoma in a dog. Australian Veterinary Journal, New South Wales, v. 77, n. 3, p. 163-165, 1999.

BUSIC, N.; BAGATIN, M.; BORIC, V. Tongue flaps in repair of large palatal defects. J ournal of Oral Maxillofacial Surgery, Zagreb, v. 18, p. 291-293, 1989.

Ciência Rural, v. 33, n. 1, jan-fev, 2003. 
DIXIT, U. B. et al. Periosteum in regeneration of palatal defects. Celft Palate Craniofacial Surgery, Zagreb, v.18, p.291-293, 1989.

FUTRAN, N.D.; HALLER, J.R. Considerations for free-flap reconstruction of hard palate. Archives of Otolaryngology Head Neck Surgery, Atlanta, v. 125, p. 665-669, 1999.

HARVEY, C.E. Palate defects in dogs and cats. Compendiun on Continuing Education for the Practicing Veterinarian, Lawrenceville, v. 9, n. 4, p. 404-418, 1987.

HARVEY, C.E.; EMILY, P.P. Oral surgery. In: Small animal dentistry. Baltimore : Mosby, 1993. Cap. 10, p.312377.

HEAD, K.W. Tumors of the alimentary tract. In: MOULTON, J.E. Tumors in domestic animals. 3 ed. Berkeley : University of California, 1990. Cap. 8, p.347-435.

HOSKINS, J.D.; DIMSKI, D.S. O sistema digestivo. In: HOSKINS, D.J. Pediatria veterinária - cães e gatos do nascimento aos seis meses. 2 ed. Rio de Janeiro: Interlivros, 1997. Cap. 10, p.120171.

LAMMERDING, J.J.; HOWARD, D.R.; BLOOMBERG, M.S. Repair of an acquired oro-nasal fistula in a dog. Journal of American Animal Hospital Association, Denver, v. 12, n. 1, p. 64-69, 1976.
NELSON, A.W. Sistema respiratório superior. In: SLATTER, D.H. Manual de cirurgia de pequenos animais. São Paulo : Manole, 1998. v. 1 , cap.52, p.884-935.

ORTON, E.C.; PARK, R.D. Avaliação do paciente portador de afecção respiratória, can didato a procedimento cirúrgico. In: SLATTER, D.H. Manual de cirurgia de pequenos animais. São Paulo : Manole, 1998. v.1, cap.51, p.873-883.

SAGER, M.; NEFEN, N. Use of buccal flaps for the correction of congenital soft palate defects in three dogs. Veterinary Surgery, Davis, v.27, n. 4, p. 358-363, 1998.

SALISBURY, S.K. Cavidade oral. In: BOJRAB, M.J. Técnicas atuais em cirurgia de pequenos animais. São Paulo : Roca, 1996, cap.10, p.144-176.

SKOOG, T. The use of periosteal flaps in the repair of primary palate. Cleft Palate Journal, Laramie, v.2, p.332-339, 1985.

TOUFFUT, G. et al. Tratamento cirúrgico das fissuras palatinas de origem acidental no gato. A Hora Veterinária, Porto Alegre n. 72, p. 16-17, 1993.

WITHROW, S.J. Tumors of the gastrointestinal system: cancer of the oral cavity. In: WITHROW, S.J.; MacEWEN, E.G. Small animal clinical oncology. Philadelphia : Saunders, 1996. Cap.18, p.227-267. 